\title{
A Systematic Search for TeV Halos associated with known pulsars
}

\author{
Andrew J. Smith* for the HAWC Collaboration ${ }^{\dagger}$ \\ University of Maryland, College Park \\ E-mail: asmith8@umd.edu
}

The HAWC gamma-ray observatory has detected very high energy gamma-ray emission from numerous PWNe. The radiation originates from pulsar driven winds accelerating electrons and positrons, which propagate into the local medium and up-scatter photons. Notable among the HAWC detect ions are Geminga and PSR B0659+1414, which exhibit large extended, low energy density 'halos' reaching 10s of pc away from the central source. In this analysis, we seek to understand if these halos are a ubiquitous feature, or depend on the specific environments or other characteristics of the pulsars. Using the Geminga pulsar halo as a template and the ATNF catalog as a target list, we performed a systematic search for $\mathrm{TeV}$ emission from known young and middle-aged pulsars. By assuming Geminga-like features, if a pulsar's distance and spin-down power are known, we can predict both the flux and extent and compare it to the measurements by HAWC.

36th International Cosmic Ray Conference -ICRC2019-

July 24th - August 1st, 2019

Madison, WI, U.S.A.

\footnotetext{
* Speaker.

${ }^{\dagger}$ HAWC Author List: https://www.hawc-observatory.org/collaboration/icrc2019.php
} 


\section{Introduction}

Pulsars, rapidly rotating neutron stars with strong magnetic fields, readily radiate energy through charged particle acceleration for at least about a million years from their formation. This emission comes in the form of pulsed emission detected from Radio to $>\mathrm{TeV}$ gamma rays beamed from their rotating magnetic poles due to the misalignment of the magnetic field and the rotations axis. Additionally, the pulsar driven winds accelerate charged particles forming a nebula (pulsar wind nebula, or PWNe) surrounding the pulsar, which is the site of charged particle acceleration to well beyond $100 \mathrm{TeV}$. Bright young pulsars are commonly detected in VHE gammas rays with luminosities strongly correlated with pulsar's spin-down power. Additionally, the VHE emission has been observed to be extended by $\approx 10$ s of pc, often well beyond the extent of the PWNe.

In 2017, the High Altitude Water Cherenkov (HAWC) collaboration showed that the TeV nebulae surrounding Geminga and B0656+14 [1] had extension well beyond that of the PWN. This result is easily interpreted as an energy dependent diffusion of electrons from the acceleration site near the pulsar into the ISM. The gamma rays result from inverse-Compton scattering of the VHE electrons off of ambient photons. In this model, the gamma-ray flux has an energy dependent extension that is governed by the diffusion characteristics in the vicinity of the nebula. Therefore, measuring the extension of these PWNe allowed for an indirect measure of the diffusion coefficient for cosmic rays in the vicinity of the pulsar, which was found to be roughly 2 orders of magnitude lower than the indirect measures from the galaxy derived from cosmic-ray abundance measurements.

At about the same time as the publication of the HAWC result, Linden et al [2] suggested that $\mathrm{TeV}$ nebula, which the authors call "Halos" to distinguish them from the much smaller PWNe observed mainly in x-rays, are a ubiquitous feature of middle-aged pulsars and their presence should be completely predictable from the pulsars age, spin-down power and distance. Using Geminga as a template he was able to predict the flux and extent of other middle-aged pulsars, both seen by HAWC and predictions for the flux of PWNe that have not yet been detected by HAWC.

In this paper, we search for pulsar halos below the nominal $5 \sigma$ discovery threshold of HAWC. The analysis considers ATNF pulsars with known distances as target candidates and a model for flux and extent based on the observed flux and extension of Geminga.

\section{The HAWC Gamma-Ray Observatory}

The High Altitude Water Cherenkov (HAWC) observatory is a wide-field continuously operating gamma-ray observatory located at an elevation of 4100m a.s.l. near the Sierra Negra volcano in central Mexico. HAWC is constructed from an array of $3007.3 \mathrm{~m}$ diameter water tanks covering roughly $22,000 \mathrm{~m}^{2}$. Each tank contains $4.5 \mathrm{~m}$ of water and is instrumented with 4 upward facing PMTs anchored to the bottom. Surrounding the central detector is a newly installed array of 350 "outrigger" tanks. The outriggers consist of small 2000 liter tanks, each instrumented with a single PMT and are designed to extend the effective collection area of HAWC by about a factor of 4 for high energy observations.

In contrast to imaging atmospheric Cherenkov telescopes (IACT), HAWC is able to operate continuously, day and night and in all weather and has a wide field of view, limited only by the 
attenuation of particles in the atmosphere as it thickens with increasing zenith angle, but we generally characterize the instrument's field of view as $90^{\circ}$ total or $60^{\circ}$ with nearly full sensitivity. Additionally, HAWC's energy threshold is generally higher than that of IACTs, since secondary shower particles from high energy cascades need to reach observation level to be detected, whereas IACTs can detect particles that range out in the atmosphere through the Cherenkov light that they emit.

Unlike most previous extensive air shower arrays, HAWC uses a thick layer of water as a detector medium. This permits us to detect both the electron and gamma-ray components of the cascade efficiently and to make calorimetric measurements of the energy deposited in each tank, a feature that is critical to the effectiveness of the cosmic-ray background detection, which at high energies can be well in excess of $99 \%$. The angular resolution of HAWC ranges from about $0.15^{\circ}$ to $1.0^{\circ}$ depending on the energy and the zenith angle and energy of the gamma ray. For details on the performance characteristics of the HAWC detector see our paper on the high-significance detection of the Crab [3].

The data set used in this analysis consists of 1128 days of data collected between November 30, 2014, when HAWC began collecting science-quality data, to May 23, 2018. This 3.5 year data sample is significantly larger than the on used when constructing the 2HWC catalog [4].

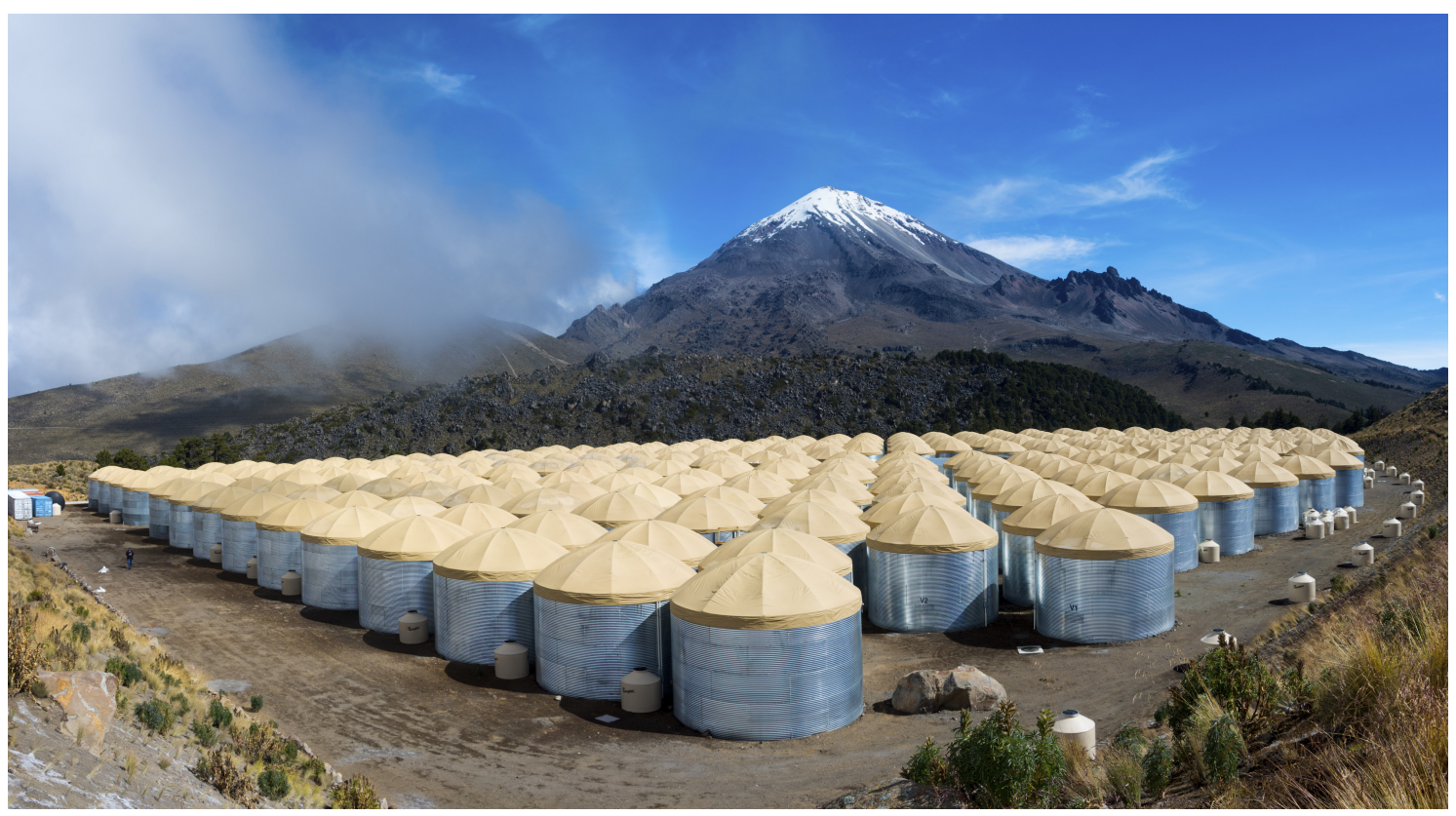

Figure 1: The HAWC site during the deployment of the outrigger array. The central dense tank array is showing with a space array of much smaller outrigger tanks around the perimeter of the platform.

\section{Pulsar Candidates}

We selected a list of candidate pulsar targets from the version 1.60 of the ATNF catalog [5] of detected pulsars, which was updated in May 2019. The catalog in total includes 2702 entries, mainly from radio-detected pulsars, and is the most comprehensive list of known pulsars available. 
Only pulsars with measured distances, age and spin-down power $(\dot{E}$ were considered. Of these, we further restricted the list to include only "young" and "middle-aged" pulsars with ages less than $10^{6}$ years. As pulsars age, and their fields diminish, they are expected to cease efficient particle acceleration.

Additionally, we only considered pulsars that transit HAWC's field of view. For this analysis, we define this region as the declination range, $\delta \in\left[-16^{\circ},+54^{\circ}\right]$, transiting within $35^{\circ}$ of the HAWC zenith. In total, there are 158 Pulsar Halo candidates.

\section{Analysis Technique}

We use the Geminga pulsar as a template for all pulsars of all ages, distances and spin-down power. To this end, the procedure is to fit the flux and extent of the Geminga pulsar halo as observed by HAWC and then scale the fit to predict the extent and flux of the other 157 candidates assuming that the flux scales linearly with $\dot{E}$ and like the inverse square of the distance. To optimize this analysis, it is critical to properly the predict extent of each pulsar when preforming the fits. HAWC's angular resolution in many cases is much smaller than the predicted extent of the pulsar Halo, so each pulsar must be treated as an extended source with an extension that is predicted by the distance.

In general, the spectrum as a function of the extent depends on the diffusion characteristics in the vicinity of the pulsar and each candidate could be fit to an extended emission model as was done in the paper exploring in the vicinity of Geminga. However, for this analysis, a simpler approach was employed, where the extent of Geminga was fit to a 2D Gaussian and the spectrum is assumed to be a power law with an index of -2.5 . This simplified approach is justified by the observation that the significance of the excess of Geminga is no found to be diminished when fit to a 2D Gaussian instead of a diffusion model. In other words, the shape is sufficiently Gaussian-like that it either fit will perform adequately when the goal is discovery of sub-threshold halos, even though further studies that work to understand the shape of the halos requires a more detailed model.

Using Geminga as a template is only appropriate for middle-aged pulsars, that is, those that are old enough that they have grown to their full size. This is where the age is large compared to the lifetime of very high energy electrons ( $20 \mathrm{ky}$ ) and the halo can be thought of as being in a stable steady state. Younger pulsars will be seen as more compact and less luminous than expected by scaling Geminga as a template, since they have not yet grown to their steady-state size and their electron injection rate has not come into balance with the electron extinction rate due to interactions with target photons in the ISM. For these reasons, most authors limit their halo searches to pulsars with ages greater than $50 \mathrm{ky}$ or $100 \mathrm{ky}$.

For this work, we chose to be comprehensive and to attempt to include as many pulsars as possible and would like to include all pulsar regardless of age. We note that many bright pulsars are young, including MGRO J2019+37 and J1908+06 with ages between 10ky and 20ky and even the Crab can be regarded as an infant Halo with an age of only $1 \mathrm{ky}$. To this end, we use a simple model for the extension and flux of pulsars as a function of age. We assume that the flux and age asymptotically approach the steady-state with a characteristic time scale equal to the electron lifetime, assumed to be 20ky. The predicted luminosity increases linearly with time as it scales with the size of the population of injected electrons and the size is assumed to scale as $\propto \sqrt{\text { time }}$ as is 
expected for diffusive processes. Figure 2 shows the predicted evolution function for as a function of the pulsar age. We would like to emphasize that the purpose of this phenomenological modeling is not to dynamically model for the evolution of young pulsars, it is simple approximation that gives what is likely a sensible result for young pulsars and could potentially aid in the discovery of halos surrounding young pulsars.
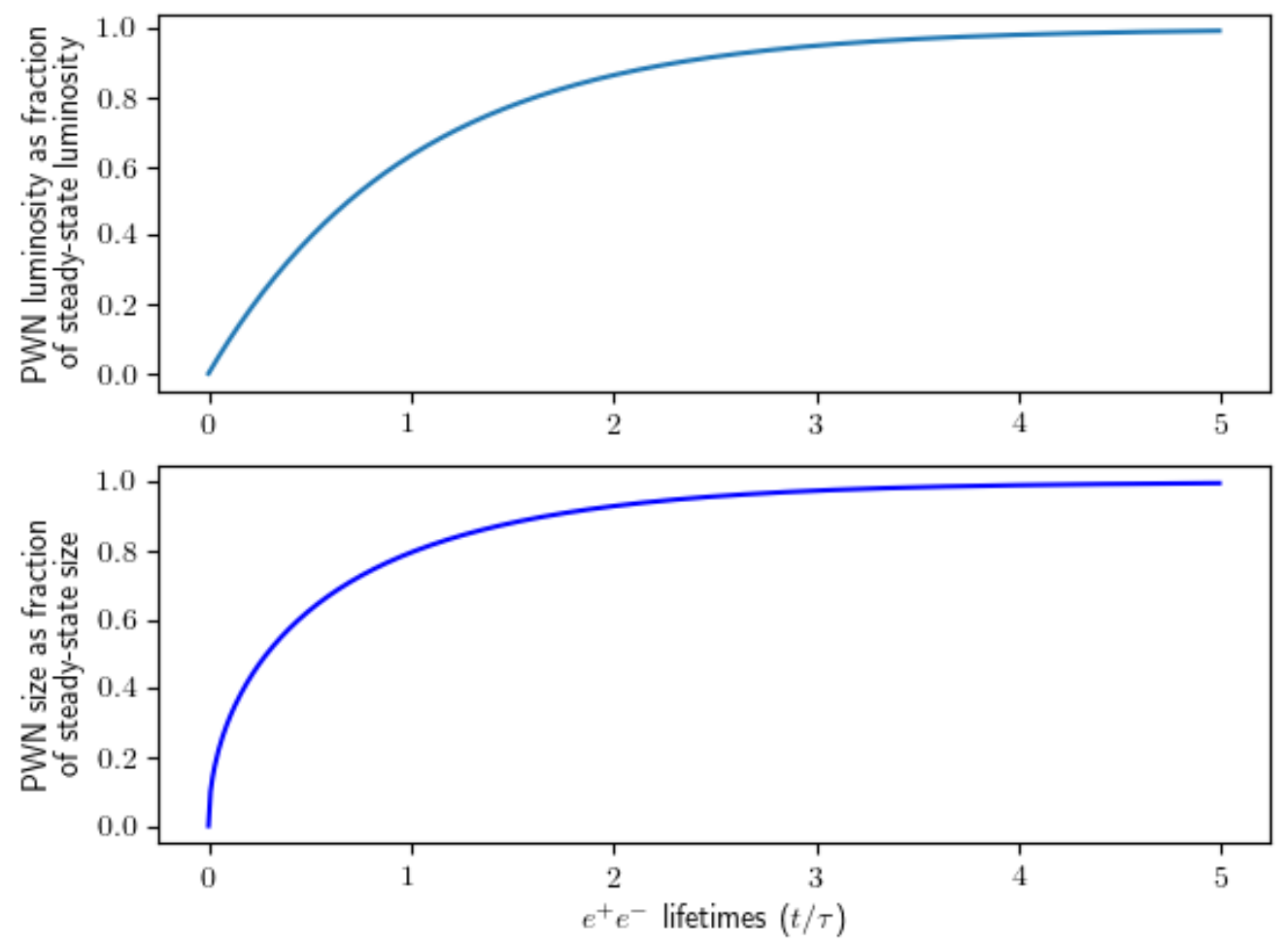

Figure 2: The modeled evolution of flux and size of pulsar halos vs age. $t$ is the pulsar age and $\tau$ is the lifetime of $e^{+} e^{-}$. In this analysis, $\tau$ is assumed to be $20 \mathrm{ky}$.

We fit the gamma-ray excess at the position of Geminga to a 2D Gaussian with $\sigma=1.78^{\circ}$. The TS of the fit is 312. Geminga been has a measured by parallax [7]to have a distance from earth of $250_{-62}^{+120} \mathrm{pc}$, and a dispersion measure estimated distance of $190 \mathrm{pc}$ [8]. As these 2 measurements are statistically compatible, we choose to use the dispersion measure as the baseline for this analysis.

Most of the pulsar candidates in the HAWC field lie within the galactic plane with galactic longitude less than $50^{\circ}$. This region is highly confused with large areas of gamma-ray excess. These confused regions, while the emission may in total be dominated by pulsar halos, require multi-source modeling to separate the emission of the pulsar halo from other gamma-ray sources. Figure 4 shows the "inner galaxy" region as seen in the HAWC data with the positions of the pulsar halo candidates with the size of the circle indicating the $68 \%$ containment radius for gamma rays as predicted from the model. In this paper, we exclude the galactic plane region, $0^{\circ}<l<$ : $100^{\circ} \cap-4^{\circ}<b<4^{\circ}$, which greatly reduces the number of source candidates. Additionally, we do not report on very weak and distant sources and require that the predicted flux be at least $1 \%$ that of 


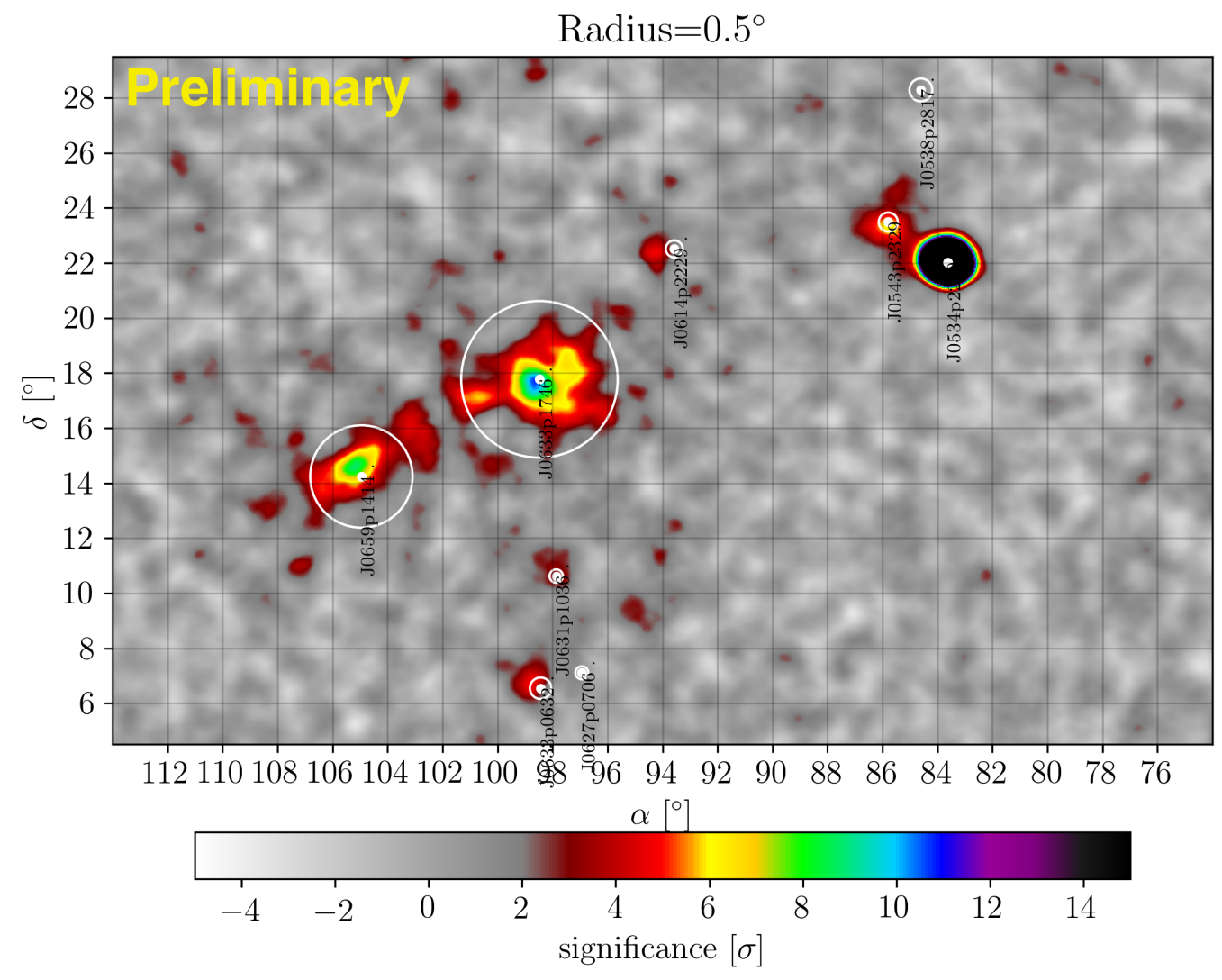

Figure 3: The wide region in the vicinity of the Crab, Geminga and B0656+15. The white circles show the predicted location and extent (68\% containment) predicted from the model.This figure has been smoothed with a Gaussian with sigma $=0.5^{\circ}$.

Geminga. In total, we report on 13 pulsar halo candidates, where 6 of are previously detected $\mathrm{TeV}$ catalog [6] sources and 7 are candidates that have not previously been detected in the VHE band.

\section{Results}

We applied our standard maximum likelihood fitting technique to each source candidate. The $\mathrm{TeV}$ emission is assumed to be centered at the location of the pulsar. It has been noted [9] that for low energy emission, the proper motion of the pulsar may cause the emission to be spread out and not centered at the pulsar position, but for this analysis, the effect is likely small due to the relatively short lifetime of VHE radiation. Therfore, we ignore proper motion in this analysis and take the current pulsar position as the center of the halo. Since the location and the extent of each hypothetical source is not permitted to float, the resulting fit has a single DOF, the flux of the source, so the $\sqrt{T S}$ can be interpreted as the significance of the excess. 


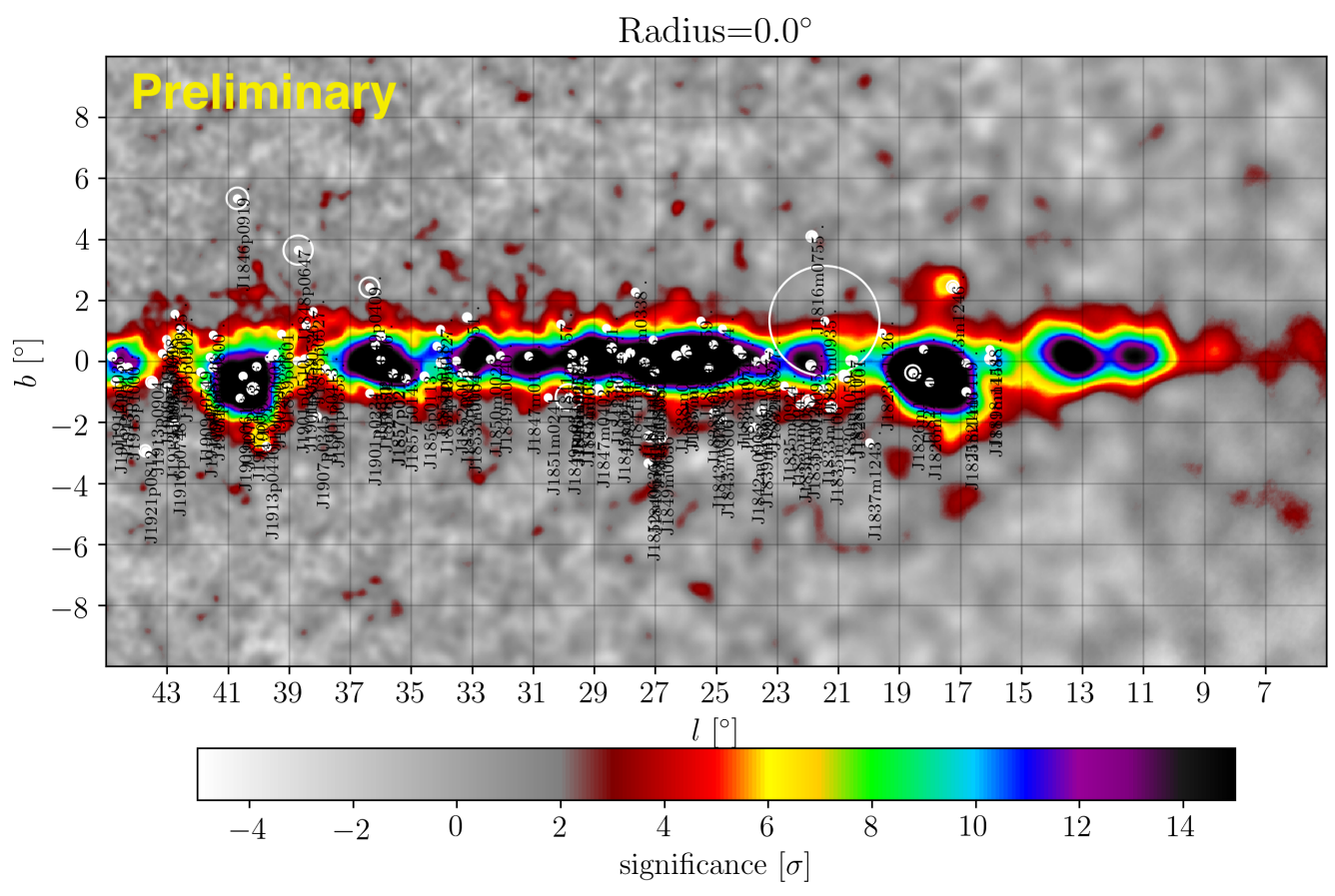

Figure 4: The Galactic plan visible to HAWC shown with overlaid white circles indicating the location and size ( $68 \%$ containment) of predicted pulsar halos. In this figure no additional smoothing was applied beyond the natural smoothing of the angular resolution of the HAWC detector.

All the bright sources are known $\mathrm{TeV}$ emitters, but several pulsars are found to have less than $5 \sigma$ excess, but an excess $>2 \sigma$, which is still quite improbable considering that only a single trial is used to examine each pulsar and there are only a small number in the final sample. The Crab, which is so young as to stretch the evolution model to its limit, is approximated as having $0.04^{\circ}$ extension ( $\sigma$ for a 2D Gaussian), which is only 3 times larger than has been observed by HESS [10] $\left(0.014^{\circ}\right)$ and under-luminous compared to the prediction by a factor of 4.5 . We regard this as a success, considering that the Crab has an $\dot{E}$ that is 4 orders of magnitude larger than Geminga, which is 350 times older.

In addition to the Crab, we report the predicted and measured fluxes of several pulsar halo candidates that are known TeV sources or very nearby. These include Geminga and PSR J0659+1414 (aka B0659+14, the pulsar purported to be the progenitor of the the Monogem SNR), which have been studied in detail using data from HAWC. We also observe an excess at high significance at the location of PSR J0633+0632, and PSR J0543+2429, which are both near recently announced HAWC sources [11, 12].

We list here some notable pulsar halo candidates that have not been reported previously as $\mathrm{TeV}$ emitters. In each case, the significance is greater than $2 \sigma$, though too small to as individual defections. 


\begin{tabular}{c|c|c|c|c|c|c|c} 
Common Name & JName & Dist $(\mathrm{kpc})$ & Age $(\mathrm{ky})$ & Size $\left.^{\circ}\right)$ & $\sqrt{T S}$ & Flux $_{\text {meas }}$ & in TeVCat \\
\hline Crab & J0534+2200 & 2.00 & 1 & 0.04 & 161.68 & 0.22 & $\mathrm{x}$ \\
Geminga & J0633+1746 & 0.19 & 342 & 1.79 & 17.66 & 1.00 & $\mathrm{x}$ \\
Monogem & J0659+1414 & 0.29 & 111 & 1.17 & 9.89 & 0.76 & $\mathrm{x}$ \\
& $\mathrm{J} 1740+1000$ & 1.23 & 114 & 0.28 & 2.99 & 0.24 & \\
& $\mathrm{~J} 0633+0632$ & 1.35 & 59 & 0.24 & 3.47 & 0.68 & $\mathrm{x}$ \\
& $\mathrm{J} 0729-1448$ & 2.68 & 35 & 0.12 & 1.74 & 1.36 & \\
& $\mathrm{~J} 0631+1036$ & 2.10 & 44 & 0.15 & 3.30 & 0.87 & \\
& $\mathrm{~J} 0538+2817$ & 1.30 & 618 & 0.26 & 0.96 & 0.39 & \\
& $\mathrm{~J} 0614+2229$ & 1.74 & 89 & 0.19 & 2.02 & 0.94 & \\
& $\mathrm{~J} 0543+2329$ & 1.56 & 253 & 0.22 & 6.29 & 3.70 & $\mathrm{x}$ \\
& $\mathrm{J} 1846+0919$ & 1.53 & 360 & 0.22 & 1.83 & 1.41 & \\
& $\mathrm{~J} 0357+3205$ & 0.83 & 540 & 0.41 & 0.13 & 0.26 & \\
& $\mathrm{~J} 0922+0638$ & 1.10 & 497 & 0.31 & 0.00 & 0.02 &
\end{tabular}

Table 1: Properties of the 13 pulsar halo candidates.

- PSR J1740+1000 is a bright middle aged (114ky) pulsar that is well off the plane. The model predicts that this source should be detected at greater than $10 \sigma$. We find a $2.99 \sigma$ excess at the candidate position with a predicted extent of $0.28^{\circ}$.

- PSR J0729+14 is a relatively young (35ky) pulsar in the outer galaxy with a rather poor declination for HAWC. At the location of this pulsar, we observe an excess of $1.8 \sigma$ and a flux measurement that is consistent with the prediction.

- PSR J0631+1036 is a fairly young (43ky) pulsar found to have a significance of $3.3 \sigma$ and a flux consistent within statistical errors of being consistent with the model. The predicted extent of the source is $0.153^{\mathrm{deg}}$.

- PSR J0614+2229 is a young (89ky) pulsar that is adjacent to supernova remnant IC443. The significance of the excess is only $2.02 \sigma$. The combination of the low significance and the confusion with IC443 make this a hard target even with significantly more data.

Figure 5 shows the relative flux from each source compared to the predicted flux. Table 1 show the results of the fits to each source for those.

\section{Conclusion}

We conducted a search of pulsar halo candidates that are outside the confused galactic plane region. In all 7 candidate pulsar locations, which have been not previously reported as $\mathrm{TeV}$ emitters were investigated. We find between $2 \sigma$ and $3 \sigma$ excesses at the locations of 3 of the 7 with good consistency with the hypothesis that many of these young and middle-aged pulsars have Gemingalike halos.

With the addition of another year of data, already collected, and upcoming improvements to the HAWC reconstruction algorithms and the extension of HAWC's high-energy sensitivity from 


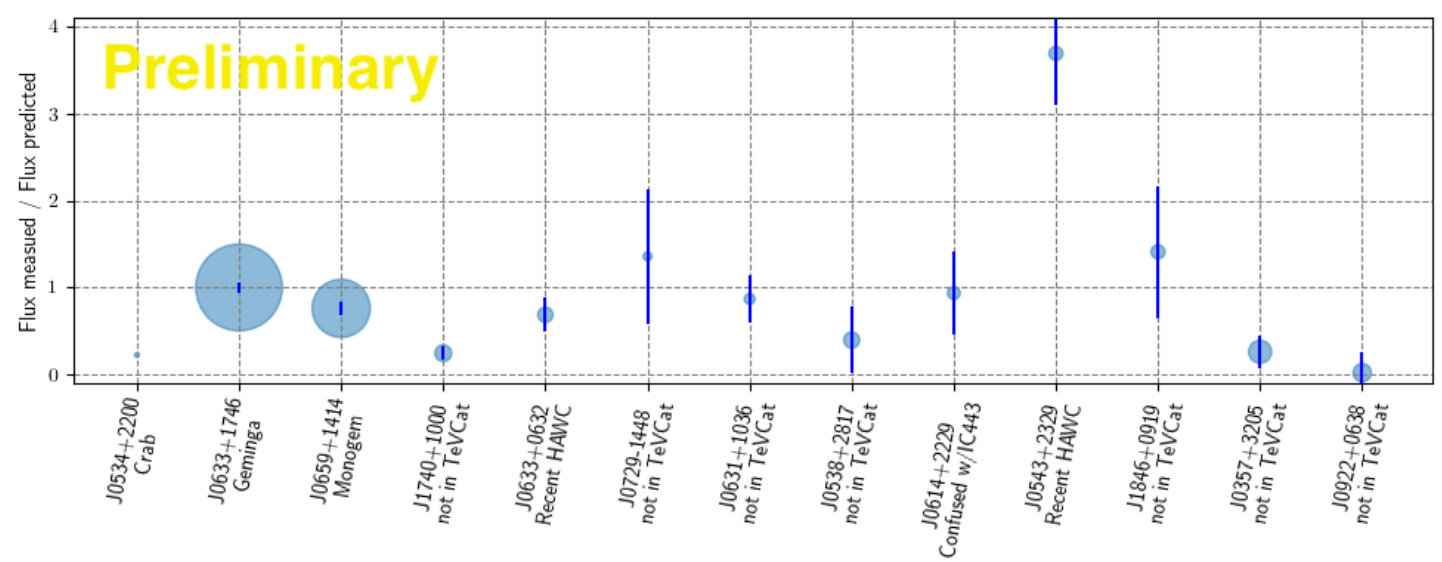

Figure 5: The ratio of measured and predicted flux for candidate pulsar halos. The size of the circle indicates the relative size as modeled.

the deployment of an outrigger array, a significant sensitivity improvement is likely and with it many new discoveries.

\section{References}

[1] A.U. Abeysekara et al, Science 358 (2017) no.6365, 911-914

[2] T. Linden et al, Phys. Rev. D 96, 103016 (2017)

[3] A. U. Abeysekara et al, The Astrophysical Journal, Volume 843, Number 1

[4] A. U. Abeysekara et al, The Astrophysical Journal, 843:39 (17pp), 2017

[5] http://www.atnf.csiro.au/research/pulsar/psrcat/

[6] http://tevcat.uchicago.edu/

[7] Faherty, J, Walter, F.M., Anderson, J. Astrophysics and Space Science, Volume 308, Issue 1-4, pp. 225-230

[8] J. M. Yao, R. N. Manchester, N. Wang, arXiv:1610.09448

[9] M. Di Mauro et al, arXiv:1903.05647

[10] M. Holler et al, Proceedings of the 35th International Cosmic Ray Conference (2017)

[11] ATel 10941; C. Riviere et al

[12] ATel 12013, C. Brisbois et al 